\title{
Wavefront error budget development for the Thirty Meter Telescope laser guide star adaptive optics system
}

Luc Gilles, Lianqi Wang, Brent Ellerbroek

Luc Gilles, Lianqi Wang, Brent Ellerbroek, "Wavefront error budget development for the Thirty Meter Telescope laser guide star adaptive optics system," Proc. SPIE 7015, Adaptive Optics Systems, 701520 (10 July 2008); doi: $10.1117 / 12.786971$

Event: SPIE Astronomical Telescopes + Instrumentation, 2008, Marseille, France 


\title{
Wavefront Error Budget Development for the Thirty Meter Telescope Laser Guide Star Adaptive Optics System
}

\author{
Luc Gilles $^{a}$, Lianqi Wang ${ }^{a}$ and Brent Ellerbroek ${ }^{a}$ \\ ${ }^{a}$ Thirty Meter Telescope Project Office \\ 1200 E. California Boulevard, Mail Code 102-8 Pasadena, CA 91125
}

\begin{abstract}
This paper describes the modeling effort undertaken to derive the wavefront error (WFE) budget for the Narrow Field Infrared Adaptive Optics System (NFIRAOS), which is the facility, laser guide star (LGS), dual-conjugate adaptive optics (AO) system for the Thirty Meter Telescope (TMT). The budget describes the expected performance of NFIRAOS at zenith, and has been decomposed into (i) first-order turbulence compensation terms (120 $\mathrm{nm}$ on-axis), (ii) opto-mechanical implementation errors $(84 \mathrm{~nm}$ ), (iii) AO component errors and higher-order effects $(74 \mathrm{~nm})$ and (iv) tip/tilt (TT) wavefront errors at 50\% sky coverage at the galactic pole $(61 \mathrm{~nm})$ with natural guide star (NGS) tip/tilt/focus/astigmatism (TTFA) sensing in J band. A contingency of about $66 \mathrm{~nm}$ now exists to meet the observatory requirement document (ORD) total on-axis wavefront error of $187 \mathrm{~nm}$, mainly on account of reduced TT errors due to updated windshake modeling and a low read-noise NGS wavefront sensor (WFS) detector. A detailed breakdown of each of these top-level terms is presented, together with a discussion on its evaluation using a mix of high-order zonal and low-order modal Monte Carlo simulations.
\end{abstract}

Keywords: Thirty Meter Telescope, Laser guide stars, Modeling, Adaptive Optics

\section{INTRODUCTION}

NFIRAOS (Narrow Field Infrared Adaptive Optics System) is the dual-conjugate, laser guide star (LGS), facility adaptive optics (AO) system for the Thirty Meter Telescope (TMT) [1-3]. Its performance requirements include $85 \%$ throughput in the I, J, H and K spectral bands, thermal radiation below $15 \%$ of the black body radiation from the telescope and atmosphere at ambient temperature, a 2 arc minute diameter field of view (FoV), and diffraction limited atmospheric turbulence compensation in the $\mathrm{J}, \mathrm{H}$ and $\mathrm{K}$ bands. The reference design $\mathrm{AO}$ configuration for NFIRAOS includes order $60 \times 60$ wavefront compensation at $800 \mathrm{~Hz}$ using a pair of conventional piezo-stack deformable mirrors (DMs) conjugate to 0 and $12 \mathrm{~km}$ range with a sheared actuator geometry, a tip/tilt platform onto which the ground-level DM is mounted, 6 LGS Shack-Hartmann (SH) wavefront sensors (WFSs) of order $60 \times 60$, and 2 near-infrared (NIR) tip/tilt $(\mathrm{TT})$ and 1 tip/tilt/focus/astigmatism (TTFA) natural guide star (NGS) SH-WFSs located within each of the NFIRAOS client instruments. According to the current plans for early light TMT scientific instrumentation [4], NFIRAOS will eventually feed 3 instruments on the telescope Nasmyth platform: IRIS (Infrared Imaging Spectrograph) with a square 10 arcsec FoV, WIRC (Widefield Infrared Camera) with a circular 30 arcsec diameter FoV, and IRMS (InfraRed Multi-Slit spectrograph) with a circular 2 arcmin diameter FoV. The performance metric for IRIS and WIRC is the residual root mean square (RMS) wavefront error (WFE), whereas for IRMS it is the PSF encircled energy (EE) within a 80 mas radius, which corresponds to a 160 mas slit width.

This paper describes the modeling effort undertaken to derive the current WFE budget for NFIRAOS feeding IRIS at a telescope zenith angle of zero degrees. Table 1 shows the top-level terms of the budget. A detailed breakdown of each of these terms is presented respectively in sections 2 to 5 , together with a discussion on the modeling and simulation tools used to evaluate them. Note that the budget evolves with improved analysis and changes to AO system designs, and that the observatory requirement document (ORD) total on-axis WFE of $187 \mathrm{~nm}$ is now met with a contingency of about $66 \mathrm{~nm}$, mainly on account of reduced TT errors due to improved telescope windshake modeling and a low read-noise detector for the three low-order NGS WFSs.

Send correspondence to lgilles@caltech.edu

Adaptive Optics Systems, edited by Norbert Hubin, Claire E. Max, Peter L. Wizinowich,

Proc. of SPIE Vol. 7015, 701520, (2008)

0277-786X/08/\$18 $\cdot$ doi: $10.1117 / 12.786971$

Proc. of SPIE Vol. 7015 701520-1 


\begin{tabular}{|c|c|}
\hline Error Term & $\begin{array}{c}\text { On-axis RMS } \\
\text { WFE (nm) }\end{array}$ \\
\hline ORD total on-axis RMS WFE & $\mathbf{1 8 7}$ \\
\hline First-order terms & 120 \\
\hline Opto-mechanical implementation errors & 84 \\
\hline AO component errors and higher-order effects & 74 \\
\hline Tip/tilt WFE at 50\% sky coverage & 61 \\
\hline Contingency & $\mathbf{6 6}$ \\
\hline
\end{tabular}

Table 1. Top-level terms of the NFIRAOS zenith WFE budget.

\section{FIRST-ORDER TURBULENCE COMPENSATION TERMS}

The fundamental first-order $\mathrm{AO}$ wavefront errors breakdown into the following terms:

1. DM fitting error, which is defined as the RMS difference between a wavefront and its least-squares best-fit (projection) onto the span of the influence functions of all DMs. The least-squares projection is restricted to a single-direction (on-axis fitting field), and performance is evaluate in that direction (on-axis performance evaluation field). This error is driven by the inter-actuator spacing and actuator geometry on the DMs.

The error has been computed using conservative (worse case) pyramidal bilinear influence functions sampled $16 \times$ more finely than the inter-actuator spacing, and multiple realizations of von Karman wavefronts generated by geometrical propagation through the 6-layer ORD turbulence profile shown in Table 4.

2. DM projection error, which is defined as the quadrature difference between a generalized, or wide-field, fitting error computed for DM actuators commanded to minimize the field-averaged RMS WFE for wavefronts across an extended FoV, and the on-axis DM fitting error defined above. RMS WFE may then be evaluated for one or several directions defining the performance evaluation field, which is not necessarily identical with the DM fitting field.

The projection error has been computed using the same realizations of the ORD turbulence profile that were used to compute the fitting error, and the fitting field was defined as $3 \times 3$ directions sampling the IRIS square 10 arcsec FoV. This error is driven by the number and ranges of the DMs.

3. WFS spatial aliasing error, which is defined as the quadrature difference between the reconstruction and fitting errors in an open-loop, noise-free, single-conjugate AO simulation using a minimum variance wavefront reconstructor, with turbulence phase screens sampled several orders of magnitude more finely than the WFS subaperture spacing. This error is driven by the WFS subaperture spacing.

4. Simulation undersampling, which is defined as the incremental (in quadrature) WFS spatial aliasing error which is unsampled with discrete turbulence phase screens (typically $1 / 64 \mathrm{~m}$ resolution).

5. Tomography error, which is defined as the quadrature difference between the residual error in an open-loop, noise-free, multi-conjugate simulation using minimum variance wavefront reconstruction, and the RSS of all the above errors. This error is driven by the guide star asterism geometry.

6. Servo lag (or bandwidth) error, which is defined as the quadrature difference between the residual errors in closed- and open-loop, noise-free, multi-conjugate simulations. This error is driven by the temporal sampling frequency and processing latency.

7. WFS measurement noise, which is defined as the quadrature difference between the residual errors in noisy and noise-free, closed-loop multi-conjugate simulations. This error is driven by the guide star signal level (photon noise), the spot size on the WFS subapertures, WFS CCD parameters (read noise) and the spot position estimation algorithm (noise propagation). 
Although each error term can be individually evaluated analytically with certain approximations using Fourier Domain (FD) techniques [5-8], simulations become useful to analyze the interactions between these errors, ensure that the system is not over-specified and maximize performance within a constrained cost. To assemble the budget, simulations are run by cumulatively including error terms in the order described above. The budget is then derived by quadrature differences. Table 2 summarizes the simulation models used for this purpose. All simulations were performed using our in-house developed Linear Adaptive Optics Simulator (LAOS) software.

\begin{tabular}{|l|l|}
\hline Optical propagation & geometrical raytracing through discrete phase screens \\
\hline Atmospheric phase screens & Periodic screens with von Karman PSD and frozen flow \\
\cline { 2 - 2 } & $64 \mathrm{~m}$ wide, $30 \mathrm{~m}$ outer scale, $1 / 64 \mathrm{~m}$ sampling \\
\hline DM actuators & Square arrays of bilinear spline influence functions \\
\hline Wavefront sensors & Subaperture-averaged wavefront gradient measurements \\
\cline { 2 - 2 } Wip/tilt removal for LGS WFSs \\
\hline WFS measurement noise & Additive, zero mean, normally distributed \\
\cline { 2 - 2 } & $\begin{array}{l}\text { LGS WFS noise statistics computed for elongated LGS and con- } \\
\text { strained matched filter spot position estimation algorithm }\end{array}$ \\
\hline Performance metrics & $\begin{array}{l}\text { Sparse minimum variance tomography, least-squares DM fitting, } \\
\text { temporal filtering with 2-frame delay }\end{array}$ \\
\cline { 2 - 2 } & Split atmospheric tomography \\
\hline
\end{tabular}

Table 2. Summary of simulation models used to derive the wavefront error budget for the first-order terms.

The split atmospheric tomography wavefront control architecture for LGS multi-conjugate AO is a generalization of the architecture for current LGS AO systems [9], and is the proposed wavefront reconstruction architecture for the NFIRAOS real-time controller (RTC) [10]. It implements two separate control loops driven independently by the LGS and NGS measurements [11]. The NGS control loop uses a noise-weighted least-squares wavefront reconstruction matrix to control 2 global TT and 3 so-called "plate scale modes". These last 3 modes consist of focus and astigmatism distributed on both DMs, which are scaled so that the wavefront propagated from the LGS asterism to the aperture-plane consists of pure TT, which is invisible to the TT-removed LGS WFSs. They are referred as plate scale modes because they introduce field dependent TT errors that magnify and distort the image at the science instrument focal plane. They also introduce field independent focus/astigmatism wavefront errors on account of the cone effect.

The LGS control loop uses an iterative, computationally efficient algorithm providing an approximate solution to minimum variance tomography, and controls all modes orthogonal to the 5 NGS modes. The benefits of the split tomography approach are a reduced coupling between the LGS- and NGS-controlled modes, a more flexible control of the TT and tilt anisoplanatism modes, and a simpler formulation of LGS tomography with reduced computation.

The fundamental AO component parameters for the NFIRAOS reference design are summarized in Table 3.

\begin{tabular}{|l|l|}
\hline Order of LGS WFSs & $60 \times 60$ \\
\hline LGS Asterism & 35 arcsec radius pentagon +1 on-axis \\
\hline Order of Ground-level DM & $63 \times 63$ (including guard band) \\
\hline Order of Upper DM & $75 \times 75$ (including guard band) \\
\hline Conjugation range of upper DM & $12 \mathrm{~km}$ \\
\hline AO Frame rate & $800 \mathrm{~Hz}$ \\
\hline Servo lag & 2 frames \\
\hline AO controller & integrator with a gain of $1 / 2$ \\
\hline
\end{tabular}

Table 3. Fundamental AO component parameters for the NFIRAOS reference design. 
DM conjugate ranges and the LGS asterism geometry were selected by trade studies, whereas the order $60 \times 60$ of sensing and correction was defined as a given to meet the TMT performance requirements using extrapolations of current technology. The $800 \mathrm{~Hz}$ frame rate was chosen based upon earlier Gemini-South multi-conjugate AO performance analyses [12]. The $25 \mathrm{~W}$ laser power per beacon corresponds to $2.5 \times$ the Gemini-South laser power requirement, which is the estimated power level needed to compensate for the increased LGS elongation (50 $\mathrm{W}$ power has now been demonstrated in the laboratory using current sum-frequency-generation sodium laser technology [13]).

The 6-layer ORD turbulence profile shown in Table 4 was used to perform the trade studies and run the AO simulations to estimate the error budget terms. The profile was derived from a 57-layer median Cerro Pachon profile obtained from the Gemini-South site testing campaign, and is characterized at zenith and a wavelength of $500 \mathrm{~nm}$ by a Fried parameter of $15 \mathrm{~cm}$, an isoplanatic angle of $2.5 \operatorname{arcsec}$ and a $29 \mathrm{~Hz}$ Greenwood frequency. The generalized isoplanatic angle for $2 \mathrm{DMs}$ at 0 and $12 \mathrm{~km}$ range is $10.5 \operatorname{arcsec}$.

\begin{tabular}{|l|l|l|l|l|l|l|}
\hline Altitudes $(\mathrm{km})$ & 0 & 2.5 & 5.5 & 7.5 & 13 & 15.5 \\
\hline Weights $(\%)$ & 65 & 17 & 6 & 3 & 7 & 2 \\
\hline Outer scale $(\mathrm{m})$ & 30 & 30 & 30 & 30 & 30 & 30 \\
\hline Wind Speeds $(\mathrm{m} / \mathrm{s})$ & 5 & 13 & 20 & 30 & 20 & 10 \\
\hline
\end{tabular}

Table 4. ORD 6-layer turbulence profile used in AO simulations.

The generalized fitting error for DM fitting and performance evaluation fields of 30 arcsec was found to vary by about $23 \mathrm{~nm}$ when the conjugation range of the upper DM was varied between 8 and $14 \mathrm{~km}$, with a minimal value at $12 \mathrm{~km}$ range. More importantly, the error was found to increase by about $48 \mathrm{~nm}$ when the inter-actuator spacing on the $12 \mathrm{~km}$ DM was increased from 0.5 to $1 \mathrm{~m}$, fully justifying the performance benefit achieved with a high-density upper DM against the cost. Regarding the selection of the frame rate and the laser power requirements, the combined error due to LGS WFS noise and servo lag was found to be minimal near $800 \mathrm{~Hz}$ for a LGS signal level of 900 photo-detection-events (PDEs) per subaperture per frame at $800 \mathrm{~Hz}$, which is the expected return from a $25 \mathrm{~W}$ laser beacon for a median sodium column density and our nominal parameters for optical and atmospheric transmission.

Table 5 shows the budget for each of the first-order terms, derived following the procedure outlined above. It is seen that DM fitting is the dominant error term of the budget, and that for a $10 \operatorname{arcsec}$ DM fitting field, the next largest terms are DM projection and tomography. The value of $30 \mathrm{~nm}$ RMS listed in the last row of Table 5 is the median value of the focus/astigmatism component of the plate scale modes, as estimated by separate sky coverage simulations [14].

\begin{tabular}{|c|c|c|c|c|c|}
\hline DM Fitting Field & On-axis & \multicolumn{2}{|c|}{10 arcsec square } & \multicolumn{2}{|c|}{10 arcsec square } \\
\hline Performance Evaluation Field & On-axis & On- & & $10 \mathrm{a}$ & csec square \\
\hline First-order terms (nm) & 120 & 127 & & 135 & \\
\hline DM actuator fitting & 76 & & 76 & & 76 \\
\hline DM projection & 0 & $\longrightarrow$ & 52 & $\longrightarrow$ & 70 \\
\hline LGS WFS aliasing & 34 & & 34 & & 34 \\
\hline Simulation undersampling & 31 & & 31 & & 31 \\
\hline Tomography & 60 & $\longrightarrow$ & 56 & $\longrightarrow$ & 50 \\
\hline Servo lag at $800 \mathrm{~Hz}$ & 21 & & 21 & & 21 \\
\hline LGS WFS noise & 39 & & 39 & & 39 \\
\hline $\begin{array}{l}\text { Focus/astig. component of plate } \\
\text { scale modes (NGS control loop) }\end{array}$ & 30 & & 30 & & 30 \\
\hline
\end{tabular}

Table 5. First-order atmospheric turbulence compensation error terms. 
In order to proceed with the evaluation of the remaining errors, LAOS has been significantly upgraded to provide simulation capabilities capturing the effects of wave optics LGS WFSs, telescope aberrations and obscurations, DM-to-WFS pupil misregistration and distortion, vertical anisoplanatism induced by 3-dimensional LGSs and DM hysteresis. These errors are discussed in sections 3 and 4.

\section{OPTO-MECHANICAL IMPLEMENTATION ERRORS}

The breakdown of the TT-removed opto-mechanical implementation errors is shown in Table 6. Please note that the dome seeing, mirror seeing, uncorrectable NFIRAOS errors and science instrument budgets are currently allocations. The telescope obscuration budget due to the primary and secondary mirrors (M1/M2) was derived as the quadrature difference between the closed loop RMS WFE for simulations including the obscured TMT pupil function sampled at a resolution of 1/64 m, and the RMS WFE obtained for an ideal unobscured circular pupil sampled at the same resolution. All simulations were done with physical optics modeling of the LGS WFSs and our nominal $60 \%$ subaperture illumination threshold, which provides optimal performance.

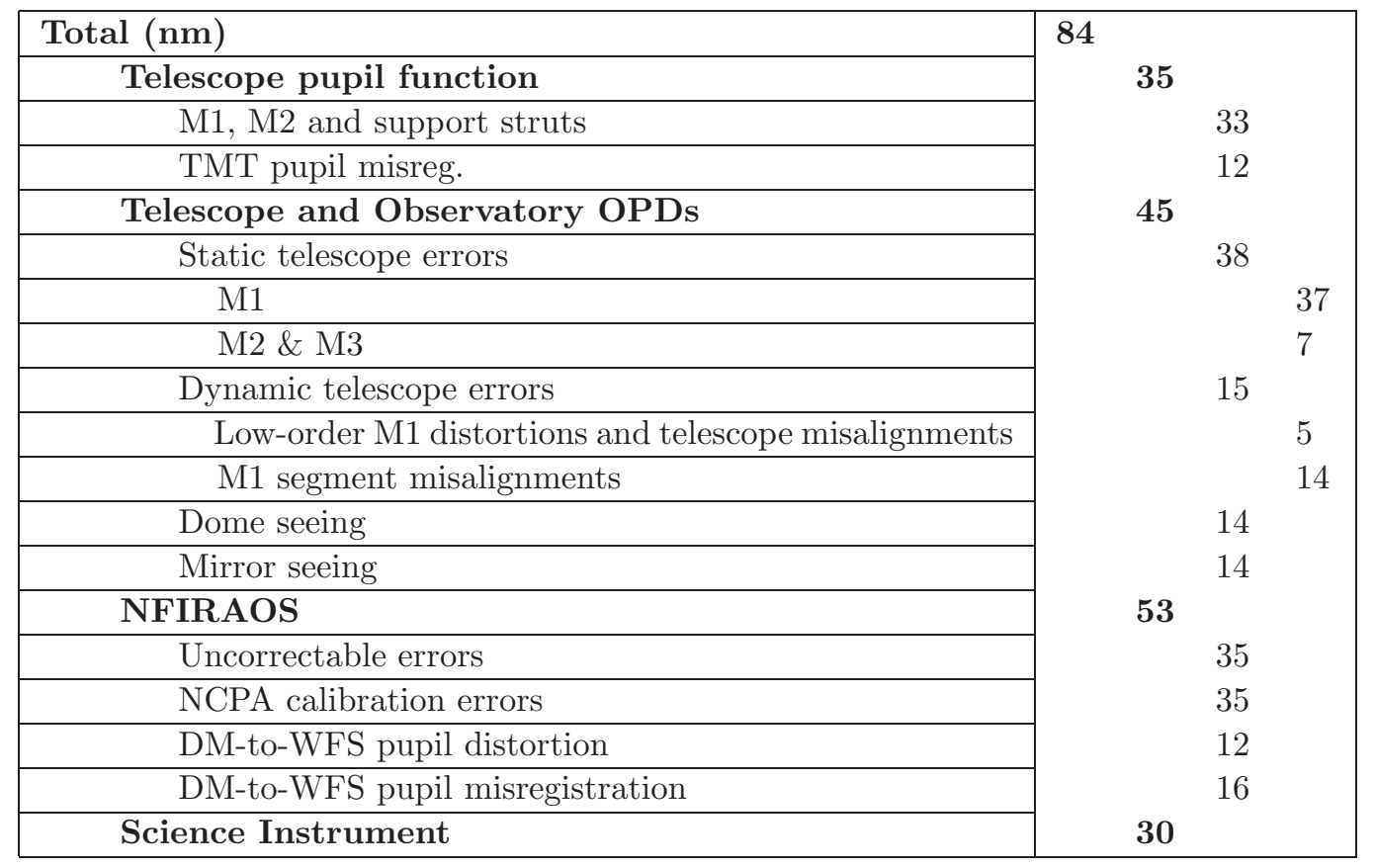

Table 6. Breakdown of opto-mechanical implementation errors.

Telescope pupil misregistration was simulated for a single-conjugate AO system by misregistering in translation a single on-axis LGS WFS and a single DM by a common amount equal to $0.3 \%$ of the pupil diameter, which is the ORD requirement on input pupil misregistration. The resulting error was found to vary between 6 and 12 nm depending upon pupil rotation angle.

Regarding the impact of static telescope aberrations, the budget was obtained as the quadrature difference of closed-loop multi-conjugate simulations with and without phase screens included for representative telescope mirror fabrication and mounting errors. Theses phase screens were generated by the Jet Propulsion Laboratory (JPL) and include segment passive support errors, segment piston/tip/tilt errors, gravity clocking and decenter, segment figuring error with warping harness correction and thermal disturbances [15].

Regarding the compensation of dynamic telescope wavefront aberrations, low-order M1 figure distortions and telescope misalignments were analyzed using a separate low-order modal simulation of NFIRAOS for the case of the $75 \%$ telescope windshake model and a $80 \mathrm{~Hz}$ NGS control loop. Optical sensitivity matrices and cross-spectra of the telescope dynamic errors provided by the Herzberg Institute of Astrophysics (HIA) and Nightsky Systems Inc. were used as input to these simulations. The dominant impact of these dynamic telescope misalignments is 
to introduce TT errors (described in section 5) and a $5 \mathrm{~nm}$ RMS WFE in the NGS modes orthogonal to global TT.

The higher-order error induced by the M1 segment misalignments caused by wind was evaluated in highfidelity LAOS simulations using wavefront maps generated for a representative $1.5 \mathrm{~m} / \mathrm{s}$ wind speed at M1 and a $1 \mathrm{~Hz}$ M1 control system bandwidth. Input disturbances were around $45 \mathrm{~nm}$ RMS and were found to be corrected to about $14 \mathrm{~nm}$.

Finally, regarding the NFIRAOS opto-mechanical error terms, mirror polishing and gravitational/thermal print-through errors of spatial frequencies greater than $1 \mathrm{cycle} / \mathrm{m}$ will be uncorrected by the DMs $(35 \mathrm{~nm}$ allocation), and non-common-path aberrations (NCPA) are assumed to be $80 \%$ correctable by calibration (35 $\mathrm{nm}$ residual of $175 \mathrm{~nm}$ errors). The budgets for DM-to-WFS pupil distortion and misregistration have been evaluated in full high-fidelity LAOS simulations using pupil distortion maps generated by HIA and translational and rotational WFS misregistration of magnitude equal to $10 \%$ of a subaperture.

\section{AO COMPONENT ERRORS AND HIGHER-ORDER EFFECTS}

The breakdown of the AO component errors and higher-order effects is shown in Table 7.

\begin{tabular}{|c|c|}
\hline Total $(\mathrm{nm})$ & 74 \\
\hline DM & 31 \\
\hline Saturation & 0 \\
\hline Hysteresis & 2 \\
\hline Influence function & 0 \\
\hline Flattening & 3 \\
\hline LGS WFS and Na layer & 55 \\
\hline Nonlinearity & 2 \\
\hline Stale matched filter & 1 \\
\hline LGS range tracking & 1 \\
\hline Tomo. point source approx. & 0 \\
\hline Rayleigh backscattering & 4 \\
\hline Atmospheric refractive index dispersion & 2 \\
\hline Chromatic anisoplanatism & 0 \\
\hline Control algorithm & 32 \\
\hline Algorithm precision & 1 \\
\hline Numerical precision & 2 \\
\hline Turbulence profile mismatch & 2 \\
\hline
\end{tabular}

Table 7. Breakdown of AO component errors and higher-order effects.

Regarding DM saturation, the NFIRAOS DM stroke requirement is $10 \mu \mathrm{m}$ peak-to-valley, which provides correction with a single DM for up to $5 \times$ the piston/tip/tilt removed wavefront error at the edge of the aperture for a von Karman turbulence profile with a $10 \mathrm{~cm}$ Fried parameter and a $60 \mathrm{~m}$ outer scale. $2 \mu \mathrm{m}$ additional stroke is available for telescope, $\mathrm{AO}$ and instrument errors. Saturation effectively never occurs in simulations with 2 DMs and the $15 \mathrm{~cm}$ Fried parameter which is used for the ORD turbulence profile. The hysteresis budget was derived from simulations with $5 \%$ hysteresis as measured by CILAS on their TMT subscale DM demo. The DM flattening error is also based upon measured performance of the subscale demo at an operating temperature of $-35 \mathrm{C}$.

Regarding LGS WFS and sodium layer errors, WFS nonlinearity was assessed by running wave optics LGS WFS simulations using a constrained matched filter spot position estimation algorithm [16] and differencing in quadrature the results obtained from simulations using an ideal, linear, geometric LGS WFS.

The NFIRAOS RTC will adapt the matched filters in real-time to compensate for sodium layer internal structure variations using line-of-sight dithering and a moderate order truth WFS $[17,18]$. These updates will 
however lag the actual variations in the sodium profile, which will induce a reconstruction error concentrated in the low-order Zernike modes whose magnitude was assessed by running LAOS simulations with a $100 \mathrm{~s}$ time lag for one particular sodium profile time history [19].

Sodium layer range variability also induces a focus error in the LGS WFS measurements, therefore causing an equal focus error in the tomographic wavefront reconstruction. This error will be measured and corrected by the TTFA NGS WFS. The residual focus error was computed analytically using the power spectral density (PSD) of the sodium layer range varations and the rejection transfer function of the focus tracking loop for a TTFA sampling rate of $100 \mathrm{~Hz}$ [20].

Also, in order to evaluate the tomographic wavefront error introduced by the point source approximation used by the reconstruction algorithm, elongated LGS images were simulated in LAOS as a sum of isoplanatic images of narrower sublayers. The resulting error was found to be negligible.

Regarding the control algorithm terms, algorithm precision is defined as the performance variation between the proposed candidate wavefront reconstruction algorithms as demonstrated in detailed simulations [11], numerical precision refers to the error induced by finite precision arithmetic (4 bytes), and turbulence profile mismatch reflects the fact that the tomography algorithm will not be precisely optimized for the true turbulence profile.

The atmospheric refractive index dispersion error arises from the fact that the AO system will slightly overcorrect atmospheric turbulence since the refractive index (and hence the optical path difference) in $J$ band is about $1.3 \%$ smaller than at the LGS wavefront sensing wavelength of $589 \mathrm{~nm}$. The resulting error is about $20 \mathrm{~nm}$ RMS for a turbulence outer scale of $30 \mathrm{~m}$, and $30 \mathrm{~nm}$ RMS for an infinite outer scale. Chromatic anisoplanatism arises from atmospheric differential refraction which causes the LGS and science beams to follow different (curved) paths through the atmosphere when projected at a non-zero zenith angle. The resulting error is zero at zenith and about $10 \mathrm{~nm}$ RMS at a zenith angle of 30 degrees.

Finally, the Rayleigh backscattering, numerical precision and turbulence profile mismatch budget terms are currently allocations.

\section{TIP/TILT WAVEFRONT ERRORS}

The ORD specifies that the atmospheric and telescope TT errors need to be corrected to a residual of 2 mas RMS (2-axis, i.e. $\sim 72 \mathrm{~nm}$ equivalent higher-order error) at $50 \%$ sky coverage at the Galactic pole. The tip/tilt performance of NFIRAOS feeding IRIS was assessed against this requirement using a separate, lower fidelity sky coverage Monte Carlo simulator with guide stars generated in the $\mathrm{J}$ band using the Besancon catalogue [14]. 500 randomly generated guide star fields following a Poisson distribution were used in the simulations. Atmospheric turbulence and the adaptive optics system were modeled in a low-dimensional Zernike space [21]. We found that this challenging TT requirement can be met with margin using (i) NIR NGS WFSs to benefit from sharpened NGS images, (ii) multiple WFSs and DMs to measure and correct tilt anisoplanatism, (iii) a woofer-tweeter control architecture for improved disturbance rejection at low temporal frequencies, and (iv) an electron bombarded detector with virtually no read-noise. The breakdown of the budget for the Armazones turbulence profile (one of the TMT candidate sites), the $75 \%$ telescope windshake model, and IRIS observations with 2 TT and 1 TTFA NGS WFSs is shown in Table 8.

\begin{tabular}{|c|c|}
\hline Total Equivalent RMS WFE (nm) & \multirow[t]{2}{*}{61} \\
\hline Telescope & \\
\hline Windshake & \multirow{3}{*}{$\begin{array}{l}18 \\
10\end{array}$} \\
\hline Other vibrations & \\
\hline Atmosphere & \\
\hline Servo lag & \multirow{3}{*}{$\begin{array}{l}14 \\
20\end{array}$} \\
\hline Anisoplanatism & \\
\hline NGS WFS noise & \\
\hline NGS WFS nonlinearity & 35 \\
\hline
\end{tabular}

Table 8. Breakdown of tip/tilt errors at 50\% sky coverage in J band at the North Galactic pole. 
The vibration and WFS nonlinearity budgets are currently allocations.

We are currently upgrading LAOS to include a sky coverage post-processing capability that will capture the effects of wave optics NGS and LGS WFSs and of the LGS split tomography, and will enable full high-order, high-fidelity sky coverage simulations with more detailed analyses of implementation effects.

\section{CONCLUSION}

High-order zonal and low-order modal Monte Carlo simulations have been conducted to evaluate and optimize the NFIRAOS wavefront error budget at zenith. Basic AO component parameters have been defined, and the error budget terms have been evaluated. Further planned analysis includes evaluating the performance variations over the expected range of operating conditions, and high-fidelity sky coverage simulations.

\section{Acknowledgments}

The authors gratefully acknowledge the support of the TMT partner institutions. They are the Association of Canadian Universities for Research in Astronomy (ACURA), the California Institute of Technology and the University of California. This work was supported as well by the Gordon and Betty Moore Foundation, the Canada Foundation for Innovation, the Ontario Ministry of Research and Innovation, the National Research Council of Canada, the Natural Sciences and Engineering Research Council of Canada, the British Columbia Knowledge Development Fund, the Association of Universities for Research in Astronomy (AURA) and the U.S. National Science Foundation.

\section{REFERENCES}

1. G.Herriot, P.Hickson, E.Ellerbroek, D.Andersen, T.Davidge, D.Erickson, I.Powell, R.Clare, L.Gilles, C.Boyer, M.Smith, L.Saddlemyer and J.-P.Véran, "NFIRAOS: TMT narrow field, near-infrared facility adaptive optics", in Advances in Adaptive Optics II, B.Ellerbroek and D.Bonaccini, eds., Proc. Soc. Photo-Opt. Instrum. Eng. 6272, 62720Q-1-62720Q-12 (2006).

2. B.Ellerbroek, S.Adkins, D.Andersen, J.Atwood, C.Boyer, P.Byrnes, R.Conan, L.Gilles, G.Herriot, P.Hickson, E.Hileman, D.Joyce, B.Leckie, M.Liang, T.Pfrommer, J.-C.Sinquin, J.-P.Véran, L.Wang and P.Welle, "Progress towards developing the TMT adaptive optical systems and their components", in Adaptive Optics Systems, N.Hubin, C.Max and P.Wizinowich, eds., Proc. Soc. Photo-Opt. Instrum. Eng. 7015 (2008).

3. G.Herriot, D.Andersen, J.Atwood, C.Boyer, P.Byrnes, B.Ellerbroek, L.Gilles, P.Hickson, B.Leckie, J.-P.Véran, L.Wang, and P.Welle, "NFIRAOS - TMT's initial adaptive optics system", in Adaptive Optics Systems, N.Hubin, C.Max and P.Wizinowich, eds., Proc. Soc. Photo-Opt. Instrum. Eng. 7015 (2008).

4. D.Crampton, L.Simard, and D.Silva, "Early Light TMT instrumentation", in Ground-based and Airborne Instrumentation for Astronomy II, I.Mclean and M.Casali, eds., Proc. Soc. Photo-Opt. Instrum. Eng. 7014 (2008).

5. F.Rigaut, J.-P.Véran and O.Lai, "An analytical model for Shack-Hartmann-based adaptive optics systems, in Adaptive Optical System Technologies, D.Bonaccini and R.Tyson, eds., Proc. Soc. Photo-Opt. Instrum. Eng. 3353, 1038-1048 (1998).

6. B.Ellerbroek, "Linear systems modeling of adaptive optics in the spatial-frequency domain," J. Opt. Soc. Am. A 22, 310-322 (2005).

7. L.Jolissaint, J.-P. Véran and R.Conan, "Analytical modeling of adaptive optics: foundations of the phase spatial power spectrum spectrum approach," J. Opt. Soc. Am. A 23, 382-394 (2006).

8. A.Tokovinin and E.Viard, "Limiting precision of tomographic phase estimation," J. Opt. Soc. Am. A 18, 873-882 (2001).

9. P.Wizinowich, D.Le Mignant, A.Bouchez, R.Campbell, J.Chin, A.Contos, M.van Dam, S.Hartman, E.Johansson, R.Lafon, H.Lewis, P.Stromski and D.Summers, "The W.M.Keck Observatory Laser Guide Star Adaptive Optics System: Overview," Pub. Astro. Soc. Pac. 118, 297-309 (2006).

10. C.Boyer, L.Gilles, B.Ellerbroek, G.Herriot and J.-P.Véran, "Update on the TMT Adaptive Optics Real Time Controller," in Adaptive Optics Systems, N.Hubin, C.Max and P.Wizinowich, eds., Proc. Soc. PhotoOpt. Instrum. Eng. 7015 (2008). 
11. L.Gilles and B.L.Ellerbroek, "Split atmospheric tomography using laser and natural guide stars," submitted to J. Opt. Soc. Am. (2008).

12. B.L.Ellerbroek and G.Cochran, "A wave optics propagation code for multiconjugate adaptive optics," in Adaptive Optics Systems and Technology II, R.K.Tyson, D.Bonaccini and M.C.Roggemann, eds., Proc. Soc. Photo-Opt. Instrum. Eng. 4494, 104-120 (2002).

13. I.Lee, G.Moule, M.P.Jalali, N.Vanasse, K.W.Groff, N.Rogers, A.K.Hankla, J.Roush, C.d'Orgeville, S.M.Adkins, Z.Prezkuta, " $20 \mathrm{~W}$ and $50 \mathrm{~W}$ guidestar laser systems update for the Keck I and Gemini South telescopes", in Adaptive Optics Systems, N.Hubin, C.Max and P.Wizinowich, eds., Proc. Soc. Photo-Opt. Instrum. Eng. 7015 (2008).

14. L.Wang, E.Ellerbroek, J.-P. Véran and J.-C. Sinquin, "The NFIRAOS tip/tilt control architecture and sky coverage for TMT," in Adaptive Optics Systems, N.Hubin, C.Max and P.Wizinowich, eds., Proc. Soc. PhotoOpt. Instrum. Eng. 7015 (2008).

15. C.Nissly, B.-J.Seo, M.Troy, G.Angeli, J.Angione, I.Crossfield, B.Ellerbroek, L.Gilles and N.Sigrist, "Highresolution optical modeling of the Thirty Meter Telescope for systematic performance trades", in Adaptive Optics Systems, N.Hubin, C.Max and P.Wizinowich, eds., Proc. Soc. Photo-Opt. Instrum. Eng. 7017 (2008).

16. L.Gilles and B.L.Ellerbroek, "Constrained Matched Filtering for Extended Dynamic Range and Improved Noise Rejection For Shack-Hartmann Wavefront Sensing", Opt. Lett. 33 (2008).

17. R.Conan, G.Herriot, O.Lardière, K.Jackson and C.Bradley, "Modeling of the Thirty Meter Telescope matched filter-based wavefront sensing", in Adaptive Optics Systems, N.Hubin, C.Max and P.Wizinowich, eds., Proc. Soc. Photo-Opt. Instrum. Eng. 7015 (2008).

18. D.Andersen, B.Ellerbroek, J.-P.Véran and L.Wang, "Negating effects from sodium profile variations for TMT: the MOR truth wavefront sensor for NFIRAOS", in Adaptive Optics Systems, N.Hubin, C.Max and P.Wizinowich, eds., Proc. Soc. Photo-Opt. Instrum. Eng. 7015 (2008).

19. L.Wang, B.Ellerbroek and L.Gilles, "Impact of sodium layer profile variability upon adaptive optics systems performance", submitted to Opt. Exp.

20. D.S.Davis, P.Hickson, G.Herriot, C.-Y.She, "Temporal variability of the telluric sodium layer", Opt. Lett. 31, 3369-3371 (2006).

21. R.Clare, B.Ellerbroek, G.Herriot and J.-P.Véran, "Adaptive optics sky coverage modeling for extremely large telescopes", Appl. Opt. 45, 8964-8978 (2006). 